\title{
Long-term functional outcomes of hearing and speech rehabilitation efficacy among Paediatric Cochlear implant recipients in Shandong, China
}

\section{Running Head-Outcomes of paediatric cochlear implantation}

\section{Article Category-Research article}

Fan Jiang ${ }^{1}$, Alimu Dayimu ${ }^{1}$, Wen-Zhe Qin ${ }^{1}$, Hannah Kuper²,

${ }^{1}$ School of Public Health, Shandong University, Jinan, China;

${ }^{2}$ International Centre for Evidence in Disability, London School of Hygiene \& Tropical Medicine, London, UK;

* Correspondence: Fan Jiang, School of Public Health, Shandong University, 44 Wen-Hua Rd, Jinan, Shandong, 250012, China. jiangfanqz@163.com; Tel.: $+86-18615511566$

\section{Declaration of Interest Statement}

The authors report no conflict of interest. 


\section{Abstract \\ Purpose}

To evaluate the auditory performance and speech intelligibility of 100 children with bilateral profound sensorineural hearing loss up to 3 years after cochlear implantation.

\section{Methods}

A cohort study was established consisting of 100 children who received cochlear implantation at Shandong Ear Nose and Throat hospital from 2012-2015. Children were examined after 1 month, 1, 2, and 3 years of implantation to assess auditory performance and speech intelligibility using standard tools. The paired Wilcoxon signed-rank test was used to assess whether the scores obtained at different testing points differed significantly. The Mann-Whitney test were utilized to examine the between-group differences (e.g. age at implantation).

\section{Results}

Three years after implantation, $60 \%$ out of 100 children reached the maximal category (7) of categorical auditory performance and 37\% achieved the highest category (5) of speech intelligibility rating. Significant improvements were found over time in categorical auditory performance category and speech intelligibility rating (from month 1 to year $1, \mathrm{P}<0.001$; from year 1 to year 2, $\mathrm{P}<0.001$; and from year 2 to year 3, $\mathrm{P}<0.001$ ). Larger improvements in auditory outcomes and speech intelligibility were observed in children with a younger age at implantation and those who received speech therapy.

\section{Conclusions}

Cochlear implantation appears to make a significant, positive contribution to the development of communication skills of young congenital and prelingually deaf children in China. These improvements continue for up to three years after implantation. Positive outcomes appear to be associated with earlier age at implantation and receipt of speech therapy.

Key words: Cochlear implant; Outcomes; Auditory performance; Speech intelligibility.

\section{Introduction}


Hearing loss is an important issue in public health worldwide[1]. The impact of hearing loss is far-reaching and, in particular, can affect the development of speech, language and cognitive skills in children[2]. Hearing loss is also an issue in China - the most populous country in the world; approximately 20 million babies are born each year in China, of whom about 60,000 are expected to have congenital hearing loss[3]. According to the 2006 National Survey of Disability, 1.49 million people were estimated to have disabling hearing loss in Shandong province in China, which included 15 thousand children under 6 years of age [4]. In 2017, the government health service reported that 2.3 thousand neonates and infants were identified with congenital or early childhood onset sensorineural deafness in Shandong province.

Cochlear implantation (CI) is an effective strategy that helps children with profound bilateral sensorineural hearing loss (SNHL) to gain the ability to hear and continue to develop language [5-7]. Since the first cochlear implant was successfully implanted in a paediatric patient in China 23 years ago, the total number of paediatric CI users has reached more than 50,000 and continues to increase every year[8]. There is no published evidence on the rates and number of paediatric CI in Shandong. However, a report from Shandong Disabled Person's Federation (SDPF) shows that in 2016, 1133 children under 4 years old from lowincome rural families received free cochlear implantation which were supported by SDPF. From September 2018, children under 6 years with profound hearing loss in Shandong province can be reimbursed $100 \%$ of the CI cost (surgery and device) through basic medical insurance schemes[9]. We believe the coverage of paediatric CI is likely to increase quickly, with improvements in medical insurance policies for children with profound hearing loss and the introduction of hospital-based universal new-born hearing screening to rural and remote areas of Shandong.

Studies have shown that CI permits significant improvement in both auditory performance and speech intelligibility $[10,11]$. The ability to improve auditory performance and communicate through speech could be considered the most important primary outcome measure of CI [12]. However, there are very few studies about the auditory and speech development of children with CI in China. Liu et al. investigated the development of auditory preverbal skills in 33 Mandarin speaking infants with CIs and found that the mean total scores of EARS auditory Questionnaire improved dramatically after cochlear implantation in the first 2 years of implant use[13]. Li et al. has followed 36 children with hearing loss (6 11 
years old) and analysed several predictors contributing to outcomes of CI, however, they didn't report the auditory performance and speech intelligibility after CI overtime[14].

With the rapid growth in the number of children with CI in China and Shandong province, there is a compelling need to study a clearly defined large group of children after cochlear implantation. The results of the study could be generalized to other young deaf children with CI in China to help maximize each child's chance of success and establish appropriate parental expectations. The objectives of this study were to evaluate the auditory performance and speech intelligibility among 100 Mandarin-speaking children with profoundly bilateral SNHL up to three years after implantation.

\section{Methods}

This longitudinal cohort study consisted of 100 profoundly deaf children who received cochlear implantation in Shandong Ear Nose and Throat (ENT) hospital from 2012-2015. This study was given ethical approval by Shandong ENT hospital (Jinan, China), and written informed consent was obtained from the parents on enrolment of their child into the study.

\section{Study Participants}

All children receiving cochlear implants at the hospital during the study period were considered for inclusion in the cohort. The age, aetiology, clinic records, hearing level and diagnosis at the time of CI surgery were obtained from medical records of children, with the authorization of parents and hospital.

Children who met the following criteria were included: (1) had no auditory brainstem response (ABR) to $95 \mathrm{~dB} \mathrm{nHL}$ stimuli before CI surgery; (2) the onset of hearing loss was at birth and of congenital aetiology; (3) had profound bilateral SNHL and underwent unilateral cochlear implantation; (4) had no other developmental disorder.

Children were excluded based on the following criteria: (1) residual hearing with pure tone audiometry (PTA) $<60 \mathrm{~dB}$ at no fewer than two frequencies $(125,250$ and $500 \mathrm{~Hz})$ or auditory steady-state response (ASSR) $<60 \mathrm{~dB}$ at 250 and $500 \mathrm{~Hz}$; (2) passed the Universal Newborn Hearing screening but was later diagnosed with hearing loss; (3) progressive hearing loss documented by regular hearing tests; (4) identifiable syndromic features have been identified by medical examination; (5) a history of conductive hearing loss.

\section{Follow-up}


All 100 children in our samples met the study criteria and were followed for three years. The participants underwent assessment of auditory and speech development and a structured interview at 1 months, 1,2 and 3 years after implantation.

\section{Data Collection}

Parental interviews were conducted to collect the following information: the birth date of children; urban or rural residence; whether universal new-born hearing screening identified hearing loss; whether one or two hearing aids were fitted before $\mathrm{CI}$ and the duration of hearing aid use; if the children received speech therapy in rehabilitation centres after CI surgery. The questionnaire was given to parents 1 month after the child received cochlear implant surgery. The parents and children returned to the hospital each year after the surgery, and the parents were then re-interviewed by an audiologist using the same question set.

The auditory and speech development of study participants were evaluated using the Chinese versions of the Categories of Auditory Performance (CAP) and Speech Intelligibility Rating (SIR) scales. The CAP scale was developed by the Nottingham group to assess the auditory performance of paediatric CI recipients [15]. It is a nonlinear hierarchical scale consisting of eight performance categories (scored from 0 "displays no awareness of environmental sound" to 7 "can use the telephone with a familiar talker"). The categorizing criteria were described in Table 1. The SIR scale was also designed by Nottingham group for studying speech production capabilities of paediatric CI recipients longitudinally[16]. It classified the children's spontaneous speech intelligibility into five categories (scored from 1 "prerecognizabe words in spoken language" to 5 "connected speech is intelligible to all listeners, and the child is understood easily in everyday contexts"). A previous study has verified the reliability of the Chinese versions of CAP and SIR [17] [18].

\section{Data Analysis}

We presented descriptive statistics of the CAP and SIR scores of the sample at the different time points. We used the Mann-Whitney test to examine the between-group differences (e.g. gender, age at implantation). The paired Wilcoxon signed-rank test was used to assess whether the scores obtained at different testing points differed significantly. All of the statistical analyses were performed using SAS 9.4. A p value of less than 0.05 was considered to show a statistically significant result.

\section{Results}


As per the eligibility criteria, the onset of hearing loss in all cases of the sample was at birth and was of congenital aetiology. All the children studied had profound bilateral deafness and underwent unilateral cochlear implantation. The sample were between 3 and 7 years of age at the time of implantation (median: 48 months).

The median CAP and SIR scores of the patients at different time periods after implantation are summarized in Figure 1. The numbers of children in each CAP and SIR category at each interval are provided in Tables 3 and 4, respectively.

Figure 1 shows the median CAP and SIR after 1 month, 1, 2 and 3 years of implant use, which indicates that the auditory performance and speech intelligible increased as the time of implant usage increased. The median CAP after 1 year of implant use (Interval-1y) was 4, which was significantly higher from 1 month of implant $(\mathrm{p}<0.001)$. The median CAP after 2 years of implant use (Interval-2y) was 6, which was significantly better than the score obtained after 1 year (Interval-1y). After 3 years of implant use (Interval-3y), the median CAP reached at category 7 , which was also significantly higher from the score obtained at Interval-2y. At Interval- 3y, 60\% out of 100 subjects reached the maximal category (7) of categorical auditory performance.

Significant improvements were also found between each of these two time points in SIR (from month 1 to year $1, \mathrm{P}<0.001$; from year 1 to year $2, \mathrm{P}<0.001$; and from year 2 to year $3, \mathrm{P}<0.001)$. Grouping SIR scores 1 and 2 as "non-intelligible speech" and scores 3, 4 and 5 as "intelligible speech" [19] shows that the proportion of children with intelligible speech increased consistently with time since implantation (Figure 2). Our data showed that, 3 years after implantation, the median SIR was 4 and $37 \%$ of our subjects achieved the highest category (5) of speech intelligibility rating.

Table 5 and table 6 consider correlates of CAP and SIR scores at the different time points. Neither gender nor urban/rural residence were related to CAP scores at any of the time points (table 5). After the first month, receipt of speech therapy and longer use of hearing aids before CI were both strongly associated with the achievement of better CAP scores. Younger age at implantation was consistently associated with the achievement of better CAP scores. In terms of SIR scores, girls and children from urban locations appeared to achieve better scores, particularly after longer follow-up (Table 6). Again, after the first month of follow-up receipt of speech therapy was related to the achievement of better SIR scores as was younger age at implantation, although use of hearing aids was not. 


\section{Discussion}

Cochlear implantation can provide substantial auditory information to children with profound hearing impairment who are unable to benefit from conventional hearing aids [20,21]. In this study, we selected CAP and SIR as the measure of auditory and speech development to allow a focus beyond clinical measures alone, to consider how cochlear implants make a real impact in children's day-to-day lives. Additionally, evaluating the progress of auditory and language level of paediatric CI users is very important to identify individuals with auditory or language development problems and suggest evidence-based rehabilitation schedules during the early stage after implantation[8,22].

The results of this study show that the children's auditory performance and speech intelligibility continued to improve over the 3-year period after implantation, with significant improvements continuing each year after implantation. The development of auditory skills and speech intelligible appears to be consistent, with all subjects making considerable gains during 3 years of implant experience. After 3 years of implant use, $60 \%$ of subjects reached the highest category of auditory performance, showing that they were able to use the telephone while $37 \%$ of subjects were fully intelligible to all listeners (i.e., SIR category 5). These scores show a substantial improvement for deaf children in auditory performance and speech, and an important step in promoting independence in their adult years.

Mandarin is a tonal language, and there are concerns that the information provided by the CI device is not optimal for tone language, hence less benefit might accrue, or might take longer to accrue [19]. It is therefore important to compare the results of our study to those previously published, from different contexts.

Beadle et al. reported from a study in UK that after 5 years of implant use, the median CAP was 6 and the median SIR was 3 [23]. Beadle et al. also noted that after 5 years of implant use, $31 \%$ out of 29 subjects reached category 7 (CAP) and $81 \%$ of 29 subjects has an intelligible speech. Camels et al. reported, in France, with a follow-up time of 3 years, $71 \%$ of implanted children has an intelligible speech with $25 \%$ which a speech intelligible rating maximum (SIR of 5) [12]. Meanwhile, a study from Taiwan by Fang followed up 84 Mandarin-speaking children with prelingually hearing loss after using cochlear implants and found that the median CAP after 3 years of implant use was 6 and the median SIR was 4 [24]. After 3 years, $21 \%$ of subjects reached the Maximal category (7) of CAP and $43 \%$ subjects reached the Maximal category (5) of the speech intelligibility rating scale. Our results on the 
whole are therefore comparable, if not better, than those previous studies. This difference may be due to differences in the sample selection, or the use of older generation of cochlear implant devices, using highly conservative audiologic criteria and less sophisticated speech strategies in previous studies[24].

The finding that the children implanted before five years old had significantly better scores on CAP and SIR scale adds to the existing body of evidence and emphasizes the need for early intervention of CI to the children with congenitally hearing loss[25-31]. With regards to the gender, our result also agrees with previous finding. Many previous studies demonstrate that girls with cochlear implants exhibit higher scores than boys on tests of speech perception[32], speech production[33], language[34] and reading[35].

Significant differences were found in our study between children grouped according to speech therapy received as this is important in improving speech-recognition performance. However, a review of current literature overall does not allow a definitive conclusion about the influence of speech therapy on the results obtained with the CI. In general, limitations in the study design of previously conducted studies, combined with relatively small samples and follow-ups of short duration, have limited the ability to make reliable conclusions. Liu et al. found that parents with high educational level in China were often sending their children to receive auditory and speech therapy in rehabilitation centers after CI surgery, which in turn promoted the acquisition of the language skills of the children [36]. Other authors, in contrast, found that the great communication benefits were achieved by subjects without routine speech therapy in 34 congenitally profoundly deaf children with CI in China[37,38].

We found that the children who had hearing aids more than 3 months before CI had significantly better scores on CAP scale. However, use of hearing aids before CI did not appear to improve the development of speech intelligibility in this study. This finding suggests that although using hearing aids before CI surgery does help children develop better auditory performance, the hearing-impaired children still require other types of stimulation to continuously improve their speech intelligibility after some years of implant use.

The outcome of paediatric cochlear implantation is characterised by its variability. Young, prelingually deaf children are a notoriously heterogeneous population with great variation in their auditory, cognitive, and linguistic maturity [39]. Following-up these groups to determine outcome is difficult to achieve in clinical practice, especially when samples of sufficient size are needed for statistical analysis. Consequently, previous studies generally included samples 
with very broad age spectrum, making it difficult to draw conclusions about effectiveness. By contrast, our study was relatively large and had narrow age range. However, our study still had several limitations which need to be taken into account when interpreting our results. First, we did not collect data about the CAP and SIR score of participants before they received CI surgery. In addition, previous studies found that five years of follow-up were needed for assessing the post-implantation development of communication ability of prelingually deaf children, while our follow-up was up to three years only. More investigations should be undertaken over a longer term (10-15 years) by the team. There were some limitations of the scales used to measure outcomes. Both the CAP and SIR scales were categorical with a ceiling effect, and both are subjectively assessed. Furthermore, neither score allows comparison to normal speech or speech perception. Language development was not assessed in the study.

\section{Conclusion}

With 3 years of CI experience, $60 \%$ of the subjects could use of telephone with known listener, and $37 \%$ were fully intelligible to all listeners. Based on the data obtained in this study on the assessed sample, it appears that cochlear implantation make as positive contribution to the development of communication skills of Mandarin-speaking congenital and prelingually deaf children in China, particularly for children who were implanted at an early age, and for those that received speech therapy.

\section{Acknowledgment}

We would like to thank all study participants for their time to be interviewed. We also thank the support of Shandong Provincial ENT hospital Affiliated to Shandong University and NHC key Laboratory of Health Economics and Policy Research (Shandong University).

\section{Reference}

1. Stevens G, Flaxman S, Brunskill E, et al. Global and regional hearing impairment prevalence: an analysis of 42 studies in 29 countries. European Journal of Public Health. 2013;23(1):146152.

2. Walch C, Anderhuber W, Köle W, et al. Bilateral sensorineural hearing disorders in children: etiology of deafness and evaluation of hearing tests. International Journal of Pediatric Otorhinolaryngology. 2000;53(1):31-38. 
3. WHO. Newborn and infant hearing screening 2009. Available from:

https://www.who.int/blindness/publications/Newborn_and_Infant_Hearing_Screening_Report . pdf?ua $=1$

4. National Bureau of Statistics. Main Data Bulletin of the Second National Sample Survey of

Disabled Persons. 2007. Available from:

http://www.stats.gov.cn/tjsj/ndsj/shehui/2006/html/fu3.htm

5. Niparko JKJ, 2010, 303(15):1498. Spoken Language Development in Children Following Cochlear Implantation. JAMA. 2010;303(15):1498.

6. Richter B, Eißele S, Laszig R, et al. Receptive and expressive language skills of 106 children with a minimum of 2 years' experience in hearing with a cochlear implant. International Journal of Pediatric Otorhinolaryngology. 2002;64(2):111-125.

7. Peixoto MC, Spratley J, Oliveira G. Effectiveness of cochlear implants in children: long term results. International Journal of Pediatric Otorhinolaryngology. 2013;77(4):462-468.

8. Lu X QZ. Auditory and language development in Mandarin-speaking children after cochlear implantation. International Journal of Pediatric Otorhinolaryngology. 2018;107:183.

9. Shandong Province Government Office. Notice of the Peoples: Government of Shandong Province on the Establishment of Rehabilitation Assistance System for Disabled Childre 2018. Available from: http://www.shandong.gov.cn/art/2018/9/30/art 2259 28657.html

10. Moog JS, Geers AE. Speech and language acquisition in young children after cochlear implantation. Otolaryngologic Clinics of North America. 1999;32(6):1127-41.

11. Bakhshaee M, Ghasemi MM, Shakeri MT, et al. Speech development in children after cochlear implantation. European Archives of Oto-Rhino-Laryngology. 2007;264(11):12631266.

12. Calmels MN, Saliba I, Wanna G, et al. Speech perception and speech intelligibility in children after cochlear implantation. Int J Pediatr Otorhinolaryngol. 2004;68(3):347-351.

13. Liu, Haihong, Jin, et al. Early auditory preverbal skills development in Mandarin speaking children with cochlear implants. 2015;79(1):71-75.

14. Su fang WHb, Ren; Zhi yin, Liu. Analysis of the factors related to the effect of speech and hearing rehabilitation after cochlear implant in prelingual deaf children. Journal of Audiology and Speech Pathology. 2015 (4).

15. Archbold S, Lutman ME, Marshall DH. Categories of Auditory Performance. Annals of Otology Rhinology \& Laryngology Supplement. 1995;166(9):312-314.

16. Allen C, Nikolopoulos T, D, O'Donoghue G. Reliability of a rating scale for measuring speech intelligibility after pediatric cochlear implantation. Otology \& neurotology : official publication of the American Otological Society, American Neurotology Society [and] European Academy of Otology and Neurotology. 2001;22(5):631. 
17. Wang Y, Pan T, Deshpande SB, et al. The Relationship Between EABR and Auditory Performance and Speech Intelligibility Outcomes in Pediatric Cochlear Implant Recipients. American Journal of Audiology. 2015;24(2):226.

18. Yu W, Tao P, Si M, et al. The Reliability Analysis of the Chinese Version of Speech Intelligibility Rate(SIR). Journal of Audiology \& Speech Pathology. 2013.

19. Wu CM, Sun YS, Liu TC. Long-term categorical auditory performance and speech intelligibility in Mandarin-speaking prelingually deaf children with early cochlear implantation in Taiwan. Clinical Otolaryngology. 2010;33(1):35-38.

20. Houston DM, Miyamoto RT. Effects of early auditory experience on word learning and speech perception in deaf children with cochlear implants: implications for sensitive periods of language development. Otology \& neurotology : official publication of the American Otological Society, American Neurotology Society [and] European Academy of Otology and Neurotology. 2010;31(8):1248.

21. Soleymani Z, Mahmoodabadi N, Nouri MM. Language skills and phonological awareness in children with cochlear implants and normal hearing. International Journal of Pediatric Otorhinolaryngology. 2016;83(4):16-21.

22. Niparko, K. J. Spoken Language Development in Children Following Cochlear Implantation. Jama. 2010;303(15):1498.

23. Beadle EA, McKinley DJNikolopoulos TP, Brough J, et al. Long-term functional outcomes and academic-occupational status in implanted children after 10 to 14 years of cochlear implant use. Otology \& Neurotology. 2005;26(6):1152-1160.

24. Hsuan-Yeh F, Hui-Chen K, Nan-Mai W, et al. Auditory performance and speech intelligibility of Mandarin-speaking children implanted before age 5. International Journal of Pediatric Otorhinolaryngology. 2014;78(5):799-803.

25. Haihong L, Xin J, Jing L, et al. Early auditory preverbal skills development in Mandarin speaking children with cochlear implants. International Journal of Pediatric Otorhinolaryngology. 2015;79(1):71-75.

26. Driver S JD. Paediatric cochlear implantation factors that affect outcomes. European Journal of Paediatric Neurology. 2016.

27. Mcconkey Robbins A, Koch DB, Osberger MJ, et al. Effect of Age at Cochlear Implantation on Auditory Skill Development in Infants and Toddlers. Arch Otolaryngol Head Neck Surg. 2004;130(5):570.

28. Nicholas JG, Geers AE. Will They Catch Up? The Role of Age at Cochlear Implantation in the Spoken Language Development of Children With Severe to Profound Hearing Loss. 2007;50(4):1048-62. 
29. Sharma A, Dorman MF, Spahr AJ. A Sensitive Period for the Development of the Central Auditory System in Children with Cochlear Implants: Implications for Age of Implantation. Ear Hear. 2002;23(6):532-539.

30. Leigh JR, Dettman SJ, Dowell RC. Evidence-based guidelines for recommending cochlear implantation for young children: Audiological criteria and optimizing age at implantation. International Journal of Audiology. 2016:1-10.

31. Dettman SJ, Dowell RC, Choo D, et al. Long-term Communication Outcomes for Children Receiving Cochlear Implants Younger Than 12 Months. Otology \& Neurotology. 2016;37(2):e82-e95.

32. Stacey PC, Fortnum HM, Barton GR, et al. Hearing-Impaired Children in the United Kingdom, I: Auditory Performance, Communication Skills, Educational Achievements, Quality of Life, and Cochlear Implantation. Ear Hear. 2006;27(2):161-186.

33. Geers A, Brenner C, Davidson L. Factors Associated with Development of Speech Perception Skills in Children Implanted by Age Five. Ear and Hearing. 2003;24(Supplement):24S-35S.

34. Geers AE, Nicholas JG, Sedey AL. Language Skills of Children with Early Cochlear Implantation. Ear and Hearing. 2003;24.

35. Geers, E. A. Predictors of Reading Skill Development in Children with Early Cochlear Implantation. Ear and Hearing. 2003;24(Supplement):59S-68S.

36. Liu H, Jin X, Li J, et al. Early auditory preverbal skills development in Mandarin speaking children with cochlear implants. International Journal of Pediatric Otorhinolaryngology. 2015;79(1):71-75.

37. Huiqun Z, Zhengnong C, Haibo S, et al. Categories of Auditory Performance and Speech Intelligibility Ratings of Early-Implanted Children without Speech Training. PLoS ONE. 2013;8(1):e53852-.

38. Amini D, Afrooz G, Daramadi PS, et al. Recognition of Disorders and Emotional Problems of Deaf Children Using House-Tree-Person and Draw-A-Person Tests in Comparison with Normal Children of Hamadan Province. Scientific Journal of Hamadan University of Medical Sciences. 2013;20(1).

39. O'Donoghue GM, Nikolopoulos TP, Archbold SM. Determinants of speech perception in children after cochlear implantation. Lancet. 2000;356(9228):466-468.

Fig. 1. The evolution of the median CAP and median SIR scores after Implantation Fig. 2 Development of speech intelligibility up to 3 years after implantation. Categories 1 and 2 are combined as unintelligible speech; categories 3,4 and 5 are combined as intelligible speech 
Table 1 Categories of Auditory Performance (CAP) criteria

\begin{tabular}{cl}
\hline Rating scale & \multicolumn{1}{c}{ Criteria } \\
\hline 7 & Can use the telephone with a familiar talker \\
6 & Understands conversation without lip reading with a familiar talker \\
5 & Understands common phrases without lip reading \\
4 & Discriminates at least two speech sounds \\
3 & Recognizes environmental sounds \\
2 & Responds to speech sounds \\
1 & Awareness of environmental sounds \\
0 & Displays no awareness of environmental sound \\
\hline
\end{tabular}


Table 2 Speech Intelligibility Rating (SIR) criteria

\begin{tabular}{cl}
\hline Rating scale & \multicolumn{1}{c}{ Criteria } \\
\hline 5 & Connected speech is intelligible to all listeners, and the child is understood easily in everyday contexts \\
4 & Connected speech is intelligible to a listener who has little experience of a deaf person's speech, and the listener does not \\
& need to concentrate unduly \\
3 & Connected speech is intelligible to a listener who concentrates and lip-reads within a known context \\
2 & Connected speech is unintelligible, and intelligible speech is developing in single words when context and lip reading \\
& cues are available \\
& Prerecognizable words in spoken language
\end{tabular}


Table 3 Number of children in each CAP category 1 month, 1-3 years after implantation

\begin{tabular}{|c|c|c|c|c|}
\hline CAP & 1 month & 1 year & 2 year & 3 year \\
\hline 0 & & & & \\
\hline 1 & 67 & & & \\
\hline 2 & 33 & 3 & & \\
\hline 3 & & 13 & 1 & \\
\hline 4 & & 44 & 13 & \\
\hline 5 & & 39 & 35 & 13 \\
\hline 6 & & 1 & 43 & 27 \\
\hline 7 & & & 8 & 60 \\
\hline
\end{tabular}


Table 4 Number of children in each SIR category 1 month, 1-3 years after implantation

\begin{tabular}{|c|c|c|c|c|}
\hline SIR & 1 month & 1 year & 2 year & 3 year \\
\hline 1 & 100 & 11 & 1 & \\
\hline 2 & & 48 & 5 & \\
\hline 3 & & 41 & 53 & 9 \\
\hline 4 & & & 41 & 54 \\
\hline 5 & & & & 37 \\
\hline
\end{tabular}


Table5 Correlates of differences in CAP between groups

\begin{tabular}{|c|c|c|c|c|c|c|c|c|c|c|}
\hline \multirow[t]{2}{*}{ Variables } & \multirow[t]{2}{*}{ Categories } & \multirow[t]{2}{*}{ No } & & & & & \multicolumn{4}{|c|}{ CAP } \\
\hline & & & \multicolumn{2}{|c|}{1 month } & \multicolumn{2}{|c|}{1 year } & \multicolumn{2}{|c|}{2 year } & \multicolumn{2}{|c|}{3 year } \\
\hline \multirow[t]{2}{*}{ Gender } & Boy & 46 & $\begin{array}{l}\text { Median } \\
1\end{array}$ & P-value & $\begin{array}{l}\text { Median } \\
4\end{array}$ & P-value & $\begin{array}{l}\text { Median } \\
6\end{array}$ & P-value & $\begin{array}{l}\text { Median } \\
7\end{array}$ & P-value \\
\hline & Girl & 54 & 2 & 0.54 & 4 & 0.24 & 6 & 0.47 & 7 & 0.61 \\
\hline \multirow[t]{2}{*}{ Community location } & Urban & 42 & 2 & & 4.5 & & 6 & & 7 & \\
\hline & Rural & 58 & 1 & 0.26 & 4 & 0.18 & 5 & 0.16 & 7 & 0.45 \\
\hline \multirow[t]{2}{*}{ Speech therapy } & No & 37 & 1 & & 4 & & 5 & & 6 & \\
\hline & Yes & 63 & 2 & 0.08 & 5 & $<0.001$ & 6 & $<0.001$ & 7 & $<0.001$ \\
\hline \multirow[t]{2}{*}{ Using hearing aids before $\mathrm{CI}$} & $>3$ months & 41 & 1 & & 5 & & 6 & & 7 & \\
\hline & $<3$ months & 59 & 2 & 0.42 & 4 & 0.001 & 5 & 0.04 & 7 & 0.01 \\
\hline \multirow[t]{2}{*}{ Age at implantation } & 36-59 months & 71 & 2 & & 5 & & 6 & & 7 & \\
\hline & $60-83$ months & 29 & 1 & 0.05 & 4 & 0.001 & 5 & $<0.001$ & 6 & 0.001 \\
\hline
\end{tabular}


Table 6 Correlates of differences in SIR between groups

\begin{tabular}{|c|c|c|c|c|c|c|c|c|c|c|}
\hline \multirow[t]{2}{*}{ Variables } & \multirow[t]{2}{*}{ Categories } & \multirow[t]{2}{*}{ No } & & & & & \multicolumn{4}{|c|}{ SIR } \\
\hline & & & \multicolumn{2}{|c|}{1 month } & \multicolumn{2}{|c|}{1 year } & \multicolumn{2}{|c|}{2 year } & \multicolumn{2}{|c|}{3 year } \\
\hline \multirow[t]{2}{*}{ Gender } & Boy & 46 & $\begin{array}{l}\text { Median } \\
1\end{array}$ & P-value & $\begin{array}{l}\text { Median } \\
2\end{array}$ & P-value & $\begin{array}{l}\text { Median } \\
3\end{array}$ & P-value & $\begin{array}{l}\text { Median } \\
4\end{array}$ & P-value \\
\hline & Girl & 54 & 1 & 1.00 & 3 & 1.14 & 4 & 0.01 & 4 & 0.03 \\
\hline \multirow[t]{2}{*}{ Community location } & Urban & 42 & 1 & & 3 & & 4 & & 5 & \\
\hline & Rural & 58 & 1 & 1.00 & 2 & 0.01 & 3 & 0.004 & 4 & 0.05 \\
\hline \multirow[t]{2}{*}{ Speech therapy } & No & 37 & 1 & & 2 & & 3 & & 4 & \\
\hline & Yes & 63 & 1 & 1.00 & 3 & $<0.001$ & 4 & $<0.001$ & 5 & $<0.001$ \\
\hline \multirow[t]{2}{*}{ Using hearing aids before CI } & $>3$ months & 41 & 1 & & 3 & & 4 & & 4 & \\
\hline & $<3$ months & 59 & 1 & 1.00 & 2 & 0.23 & 3 & 0.08 & 4 & 0.17 \\
\hline \multirow[t]{2}{*}{ Age at implantation } & 36-59 months & 71 & 1 & & 3 & & 4 & & 5 & \\
\hline & $60-83$ months & 29 & 1 & 1.00 & 2 & $<0.001$ & 3 & $<0.001$ & 4 & $<0.001$ \\
\hline
\end{tabular}




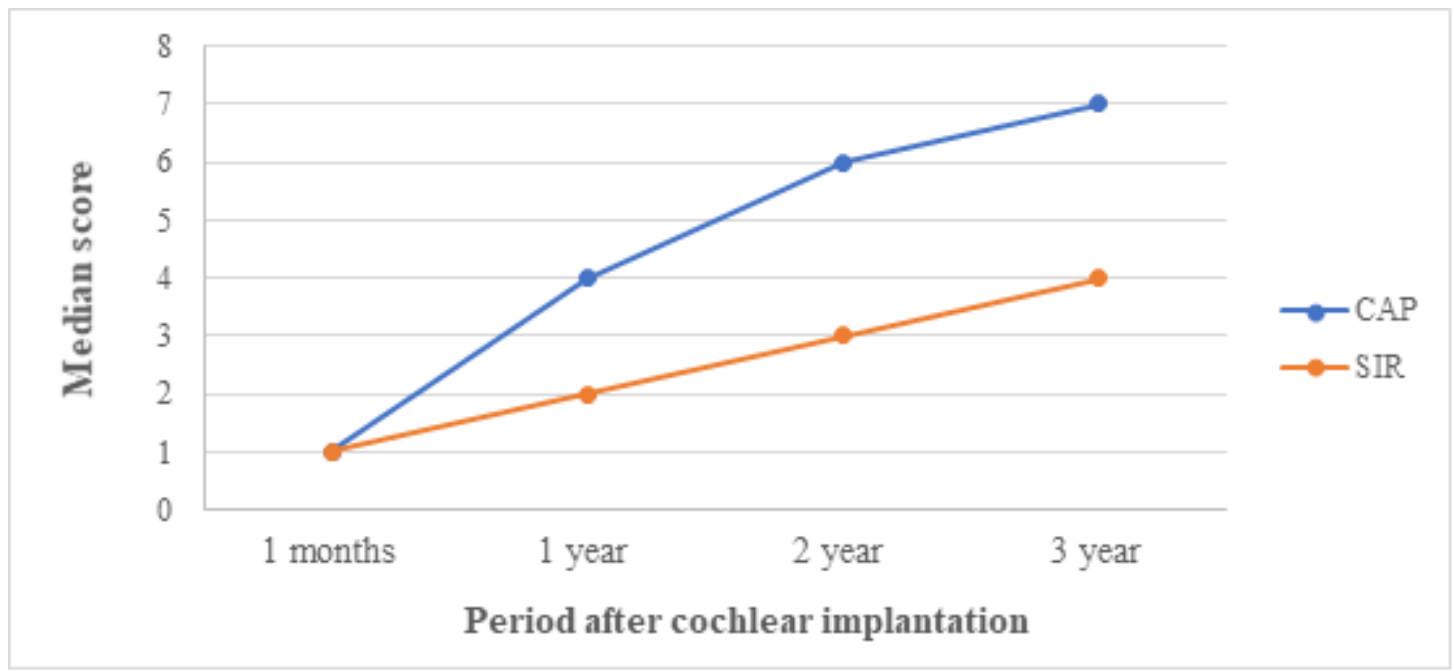

Figure 1

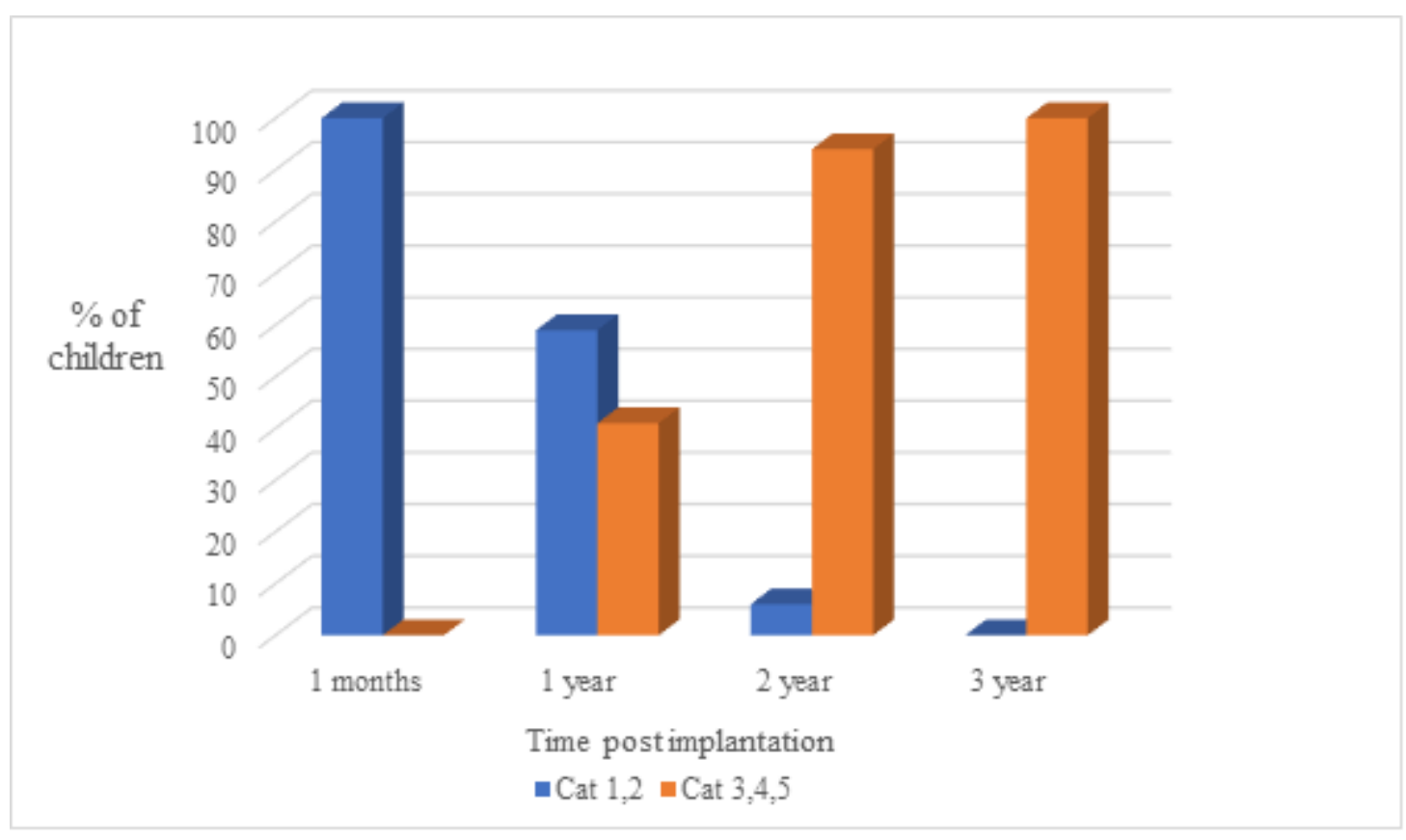

Figure 2 